\title{
The Comparison of Bone-Like Apatite Formation on Two Kinds of Bio-Active Material Surface Under the Low-Intensive Pulsed Ultrasound Waving (LIPUS) for Osseointegration
}

\author{
Masanori Kobayashi* \\ Department of Physical Therapy, Takarazuka University of Medical and Health Care, Japan
}

*Corresponding author: Masanori Kobayashi, Department of Physical Therapy, Takarazuka University of Medical and Health Care, Hanayashiki-Midorigaoka 1, Takarazuka-city, Hyogo, 666-0162 Japan

\section{ARTICLE INFO}

Received: 徰 August 24, 2020

Published: September 01, 2020

Citation: Masanori Kobayashi. The Comparison of Bone-Like Apatite Formation on Two Kinds of Bio-Active Material Surface Under the Low-Intensive Pulsed Ultrasound Waving (LIPUS) for Osseointegration. Biomed J Sci \& Tech Res 29(5)-2020. BJSTR. MS.ID.004877.

\begin{abstract}
Excellent osteoconductivity or osseointegration of biomaterial is very important for the stability of artificial joints and dental implants in clinical field.Against this background, We have already reported that the low-intensive pulsed ultrasound wave stimulation (LIPUS) could accelerate the bone-like hydroxyapatite precipitation on the bioactive material surface, which corresponds to osseointgration. The purpose of this study was to investigate the difference between the kinds of biomaterials the osseointegration enhanced by LIPUS in vitro. Hydroxyapatite $(99.9 \%)$ and Bio-active titanium were soaked in simulated body fluid under the LIPUS radiation, and accessed by scanning electron microscope and X-ray diffraction, the mass of precipitation was measured.In a result, bio-active titanium showed the more rich bone-like apatite formation on the surface than hydroxyapatite. These facts suggested that the formation of bone-like apatite might depend on the initial epitaxial nucleation growth on the biomaterial surface.
\end{abstract}

Keywords: Low-Intensive Pulsed Ultrasound Wave Stimulation (LIPUS); Hydroxyapatite; Bio-Active Titanium; Osseointegration; Simulated Body Fluid (SBF)

\section{Introduction}

Nowadays, dental implants have become more common treatment for replacing missing teeth and aim to improve chewing efficiency, physical health, and esthetics. The favorable clinical performance of dental implants has been attributed to their firm "osseointegration" as a direct contact between living bone and the surface of a load-carrying implant at the histological level introduced by Brånemark [1,2]. Considering the clinical view, more early bonding between dental implant and living bone will accelerate the onset of activity of patients soon after an operation, with subsequent good long-term results, therefore, some approach has been tried to accelerate the osseointegration or the bone bonding to HA material. Much has been learned about this concept and significant improvements on the design and surface of implants were done to eliminate the important challenges of the implant dentistry and orthopedics surgery [3-5]. Against this background, considering the efficacy of ultrasound waving for treatment of delayed union bone fractures, we have already reported that the low-intensive pulsed ultrasound waving (LIPUS) stimulation could accelerate the bone bonding ability on bioactive titanium surface [6,7] and hydroxyapatite surface [8] in vitro and in vivo studies. This fact insisted that the LIPUS is strongly expected as an excellent clinical application for the enhance of osseointegration or bone bonding of bio-active implant in dental surgery and orthopedics surgery fields.

As regarding the mechanism of this enhancement by LIPUS, our data indicated that the LIPUS could accelerate the bonelike apatite formation on the bio-conductive material surfaces, secondary this apatite layer provided the bonding with the natural bone tissue matrix. Thus, initial apatite crystallization process on material surface by ultrasound waving must be very important 
for the enhancement of Osseointegration or bon bonding ability of the material by LIPUS. On the other hand, the nucleation and crystallization are significantly depending on the surface matrix from a crystallographic point of view. Then, in this study, we assessed the differences of the bone-like apatite formation process between the two kinds of materials, hydroxyapatite, and bio-active titanium surface under the LIPUS in simulated body fluid (SBF) .

\section{Materials and Methods}

\section{Experimental Specimens}

In this experimental, specimens were prepared two kinds of biomaterials , hydroxyapatite, and bio-active titanium metal. As a hydroxyapatite material, commercial CELLYARD (CELLYARD TM pellet manufactured by HOYA, Japan) was used. Bio-active titanium specimen ( the pure bioactive titanium ) were prepared according to a following procedure. Briefly, samples of pure titanium ( $99.9 \%$ Ti ; Daido steel Co. Japan ) were cut into $3 \mathrm{~mm} \times 3 \mathrm{~mm} \times 3 \mathrm{~mm}$ cubic dice, polished and chemically treated with $\mathrm{NaOH}$ solution (concentration : 2M / l) for 2 hours reaction time. After that, these samples were heated at $600{ }^{\circ} \mathrm{C}$ for 1 hour in an electric furnace and then cooled to room temperature in the furnace for 24 hours to modify the surface.

\section{Low-intensive pulsed ultrasound waving in SBF}

Specimen were soaked in SBF and pulsed with ultrasound radiation at a period of time (Figure 1). Hanks' Balanced Salt Solution (Lonza, Switzland) was used as a SBF and maintained at $\mathrm{pH}$ over 7.0 and $37.0^{\circ} \mathrm{C}$ and replaced every three day. Ultrasound radiation was applied by using Sonic Accelerated Fracture Healing System (SAFHS:Smith \& Nephew, Memphis, TN, USA; Teijin Pharma, Tokyo, Japan). The treatment head module delivered ultrasound waves with $1.5 \mathrm{MHz}$ since waves, $200 \mu \mathrm{sec}$ signal term and spatial average intensity of $30 \mathrm{~mW} / \mathrm{cm} 2$. The radiation condition were follows; the radiation distance between the ultrasound radiator and specimens was $50 \mathrm{~mm}$, stimulation time was $20 \mathrm{~min}$ daily. As a control, the same specimens were left in SBF without ultrasound radiation under the same experimental conditions.

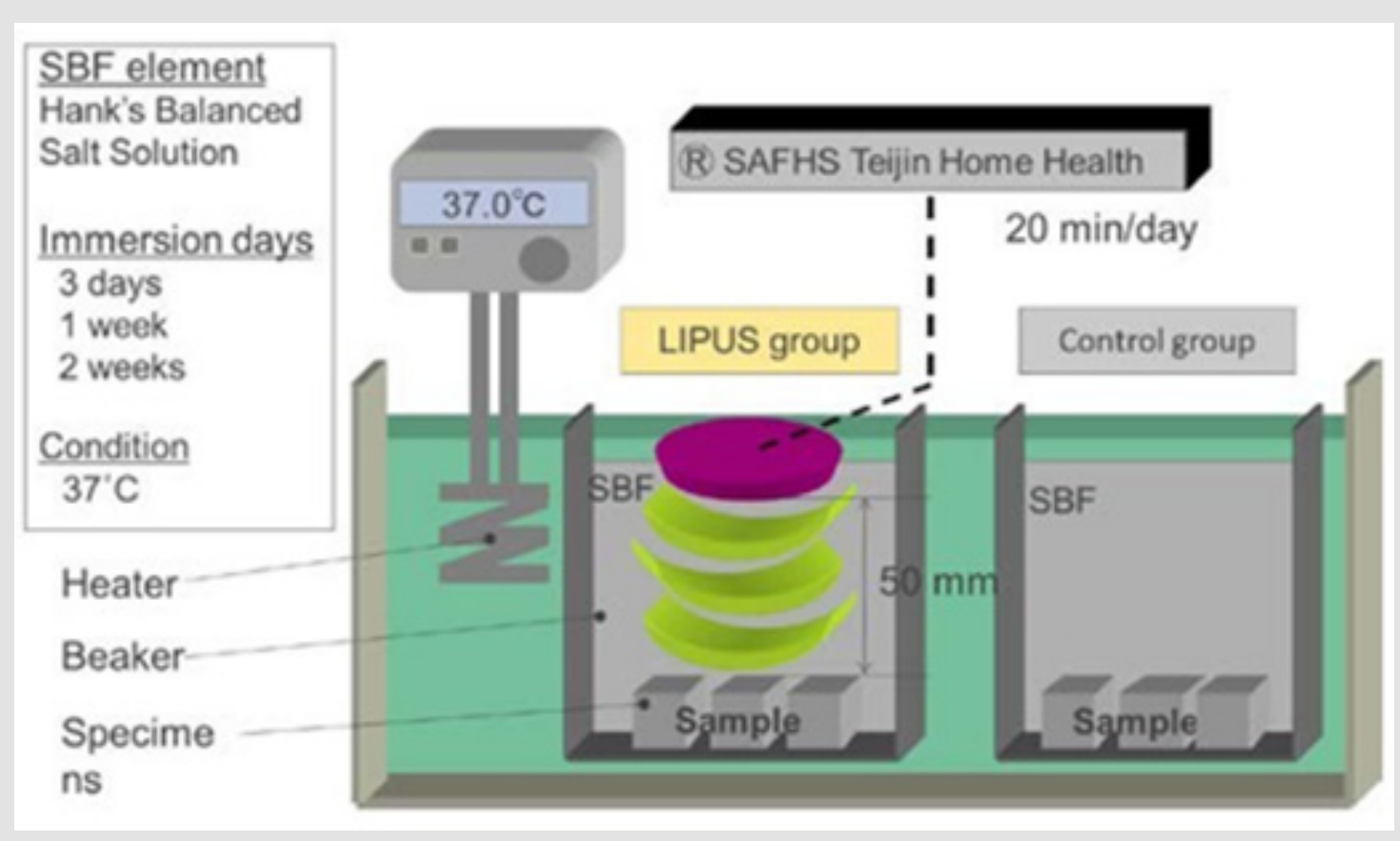

Figure 1: Schematic of the experimental method.

\section{Analysis}

After the above mentioned processes were completed, the hydroxyapatite and titanium specimens surface were subjected to scanning electron microscopy (SEM), X-ray diffraction (XRD), and these analyses were focused on the top surface, on which the ultrasound waves was directly radiated. Furthermore, to compare with the effect of ultrasound waves, the change of the specimen's mass before/after the SBF treatment was measured.

\section{Results}

\section{SEM Images}

(Figure 2 \&3) showed the SEM images of the specimen's surface after soaking for 3 days, 7 days and 2 wekks in SBF. At 3 days, ultrasound wave (LIPUS) group and Control group of the surface of the specimens can be observed a new precipitate, but there are no significant differences between the both specimens' surface. 
Next, after soaking for 1 week in SBF, both specimens showed more precipitation, LIPUS group showed increasing precipitation than the control group. After soaking for 2 weeks in SBF, LIPUS group and control group showed crystal growth covering most of specimen's surface, specimen surface of ultrasound wave group was more often observed in comparison of number of crystal particles of the base surface.

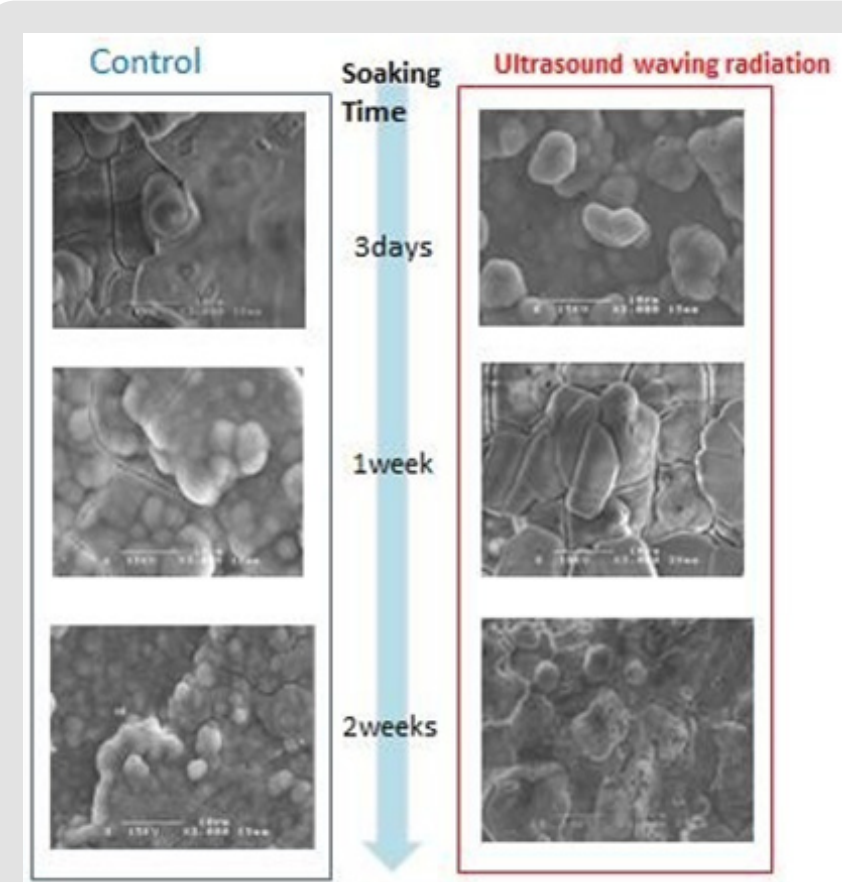

Figure 2: SEM of the Hydroxyapatite (HA) surface of specimen during the soaking in SBF for 2 weeks.

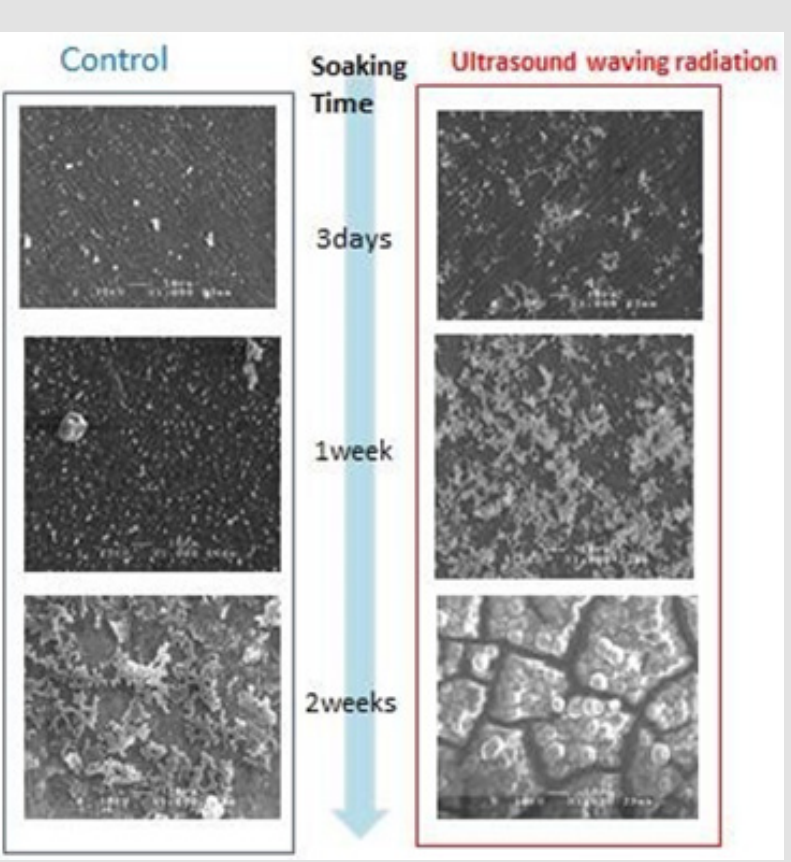

Figure 3: SEM of the bio-active titanium surface of specimen during the soaking in SBF for 2 weeks.

\section{XRD Analysis}

Figure 4 showed the XRD patterns of the precipitation on both of specimen surfaces after soaking for 2 weeks. The XRD analysis indicated the existence of a calcium-phosphate crystalline which means a bone-like apatite on surface of two kinds of specimen, some typical hydroxyapatite peaks were also confirmed.

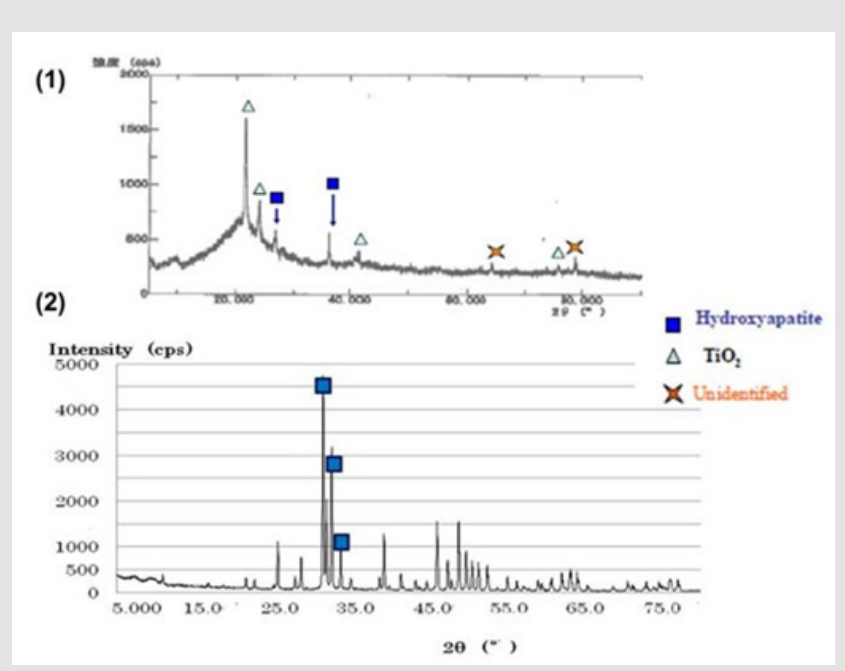

Figure 4: XRD of the specimen's surface soaked in SBF for 2weeks.

A. XRD of the Bio-active titanium surface
B. XRD of the Hydroxyapatite(HA) surface.

\section{The Mass of Precipitation}

Figure 5 showed the time dependence of mass change of bonelike apatite precipitation on specimens' surface during soaking in SBF. LIPUS group showed apparently increases of substrates compared with the control group at soaking 1 and 2weeks. In bone-like apatite on HA specimen, 3days no significant difference between LIPUS and control group was recognized. The dose of apatite precipitation on bio-active titanium surface were more than that of HA in either group.

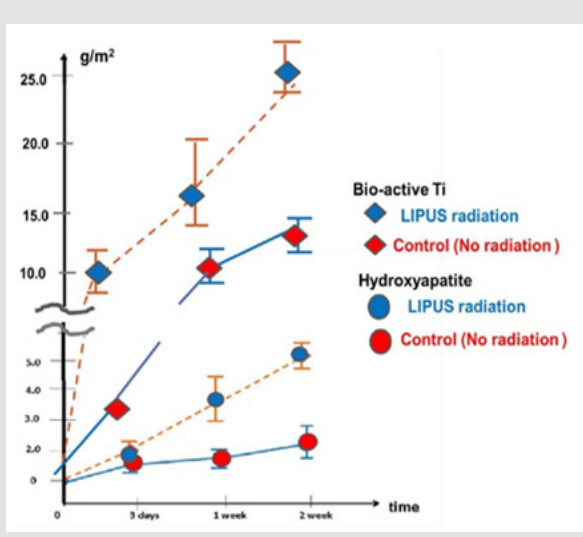

Figure 5: Time dependence of the mass of Ca-PO4 compound on the hydroxyapatite surface.Each 3 day, 1 week. And 2 week of the radiation time group. 


\section{Discussion}

The enhancement effect of LIPUS to bone-like apatite formation on bio-active materials surface from there result, it was thought that precipitate of bone kike-apatite crystal growth on specimens surface of LIPUS group, and it has been speculated that ultrasound wave accelerated to the effect of crystal growth. Kokubo et al. showed apatite deposition from SBF as evaluation method of the bioactive biomaterials in vitro, described physical deposition as the mechanism of bone formation from SBF to material surface of $\mathrm{PO}^{2-}, \mathrm{Ca}^{2+}$ and other and crystallization process [9]. This deposition process of apatite was physical and chemical changes without biological factor as well as apatite mineralization process of this experiment, therefore, it was considered that ultrasound waving with ion concentration with moderate intensity has a possibility to enhance the mineralization on the biomaterial surface. As a result, the increasing of calcium-phosphate precipitation including partial hydroxyapatite crystalline by LIPUS was confirmed. As for the mechanism, we speculate that the partial concentration and interatomic potential of the $\mathrm{Ca}$ and $\mathrm{PO}_{4}$ ions in $\mathrm{SBF}$ can be changed by ultrasound micro vibration, and accelerated to the nucleation and deposition of ions, resulting in the crystallization of apatite on material surface layer. Cavitation by ultrasound waves might also contribute to the apatite layer formation by means of the microfracture of calcium-phosphate and the circulation of new $\mathrm{Ca}$ and $\mathrm{PO}_{4}$ ions of SBF on the titanium surface. These environmental changes will accelerate further changes of interatomic potential of surrounding atoms and deteriorate the thermal stability of calciumphosphate phase versus Ca and PO4 ions. Tanaka has already reported an increase of degrees of freedom and number of crystal orientations for atomic pileup by thermal vibration concerning the relationship between the dendritic crystal and ultrasound vibration [10]. According to his report, the rapid crystallization caused by ultrasound waves is considered to enhance the nucleation process rather than the growth stage. The same ultrasound-induced crystallization has been recently reported in the metallurgy field; however, the detailed mechanism is still unclear $[11,12]$.

\section{The Comparison of Material Substrate of Bone-Like Apatite Formation}

As for the comparison of the mass of bone-like apatite between the two kinds of materials, bio-active titanium and HA, bio-active titanium demonstrated more rich formation than HA although it is difficult to evaluate the superiority of the apatite formation ability of two materials since the characteristics of material surface such as rough surface etc. we are not controlled in this study. Figure 6 shows the SEM of the two specimens' surfaces in 1day soaking in SBF. The SEM image of two materials surface at early stage in the SBF soaking showed the apparent difference of apatite crystallization on each surfaces. Bone-like apatite in titanium surface was nuclear in dendrite, while that on HA surface shows the square shape, which means the crystalline of bone-like apatite has grown in along to crystal orientation of hydroxyapatite ( c-axis ), Volmer-Weber(VW) mode in epitaxial growth. Further 2days, bonelike apatite covered the specimen surface growing in square layer. These findings suggested that the conductive crystal orientation of the initial calcium phosphate mineralization could be limited by anionic bond according to epitaxial orientation of HA ceramics. On the other hand, according to Kokubo [13], pure bio-active titanium has more excellent osteoconductivity since pure titanium has more rich modified sodium titanium hydrogel layer, which is suitable for formation of the bone-like apatite crystalline. In the view of the crystallogaphically, this modified sodium titanium hydrogel was amorphous structure, which is able to have many orientations for initial nucleation and deposition of calcium phosphate crystallization. Therefore, the seed of early apatite formation on bio-active titanium will be superior to that of HA surface.
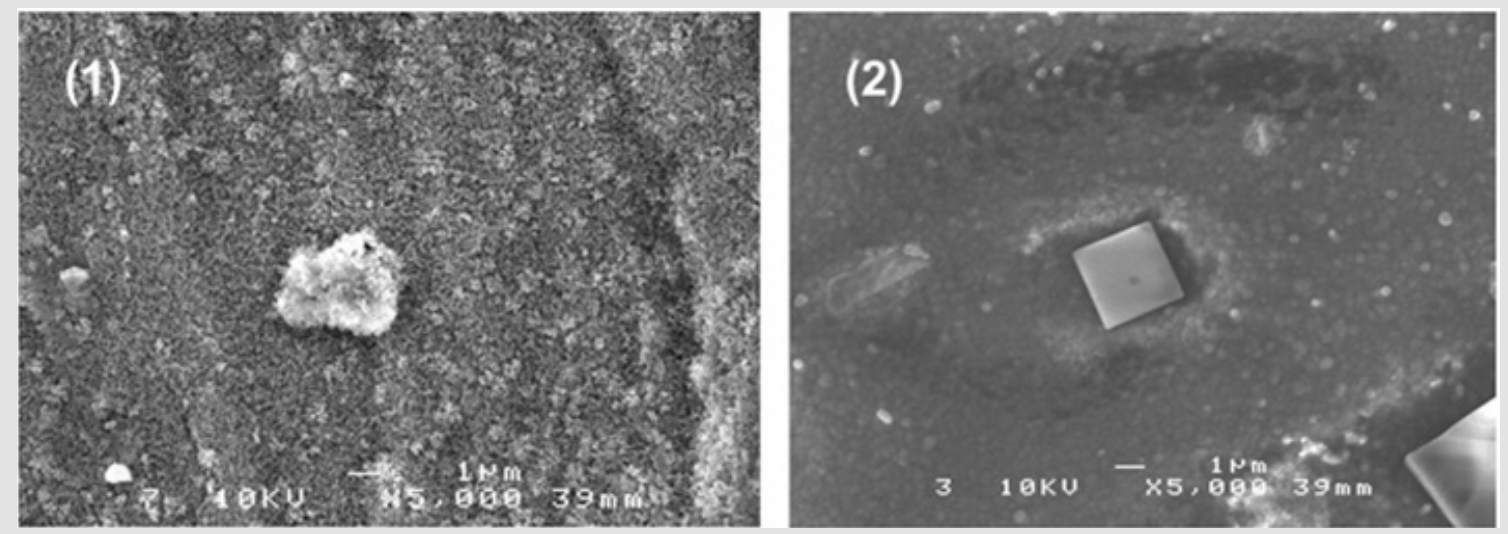

Figure 6: SEM image of the specimen's surface soaked in SBF for one day.

a) Bio-active titanium surface

b) Hydroxyapatite (HA) surface 
This result indicated that the seed of apatite formation on HA surface by LIPUS is inferior to that of bio- active titanium although hydroxyapatite is a main component which constitutes bones and teeth of an organism. In conclusion, this study suggested that LIPUS could accelerate the bone-like apatite formation on the bio- conductive material surfaces. Since this apatite layer provided secondary the bonding with the natural bone tissue matrix, initial apatite crystallization process on material surface by ultrasound waving must be very important for the enhancement of Osseointegration by LIPUS. Furthermore, comparing the crystalline of bone-like apatite on two kinds of biomaterials, bioactive titanium showed more rich bone-like apatite formation than HA. That indicated that the sodium titanium hydrogel layer of bio-active titanium surface is more suitable to initial nucleation of apatite than HA in SBF under the LIPUS as a considering the initial morphology of crystal from a crystallographic point of view. This finding suggested the possibility of modification and characterizing of implant surface as the approach to enhance the osseointgration by LIPUS.

\section{References}

1. Brånemark PI, Hansson BO, Adell R, Breine U, Lindström J (1977) Osseointegrated implants in the treatment of the edentulous jaw. Experience from a 10-year period. Scand J IPlastic and Reconstr Surg Suppl 16: 1-132.

2. Brånemark PI (1983 ) Osseointegration and its experimental background. J Pros Dentistry 50(3): 399-410.

3. Smeets R, Stadlinger B, Schwarz F, Beck Broichsitter B, Jung O ( 2016 ) Impact of Dental Implant Surface Modifications on Osseointegration. BioMed Res Inter 1- 16.

\section{ISSN: 2574-1241}

DOI: $10.26717 /$ BJSTR.2020.29.004877

Masanori Kobayashi. Biomed J Sci \& Tech Res

(C) This work is licensed under Creative

Submission Link: https://biomedres.us/submit-manuscript.php
4. Thomas KA, Kay JF, Cook SD, Jarcho M (1987) The effect of surface macrotexture and hydroxyapatite coating on the mechanical strength and histologic profiles of titanium implant materials. J Biomed Mater Res 21(12): 1395- 1406.

5. De Andrade MD, Sader MS, Filgueiras MRT, Ogasawara T ( 2000 ) Microstructure of ceramic coating on titanium surface as a result of hydrothermal treatment. J Mater Sci: Mater Med. 11 : 751-755.

6. Kobayashi M, Noda K, Tatematsu N ( 2010 ) Preliminary in vitro study on Enhancement of Bone-like Hydoxyapatite formation on bioactive titanium alloy by low-intensity pulsed ultrasound waving for early bone bonding. J Biomech Sci and Eng 5(4): 449-460.

7. Kobayashi M ( 2017 ) Study on enhancement of Osseointegration of the bio-active titanium implant by low intensive Ultrasound wave. (Part1: Simulated body fluid soaking test). Int J Biomed Eng Sci (IJBES) 4: 1-10.

8. Mukai H, Kobayashi M (2011) The Effect of Low-intensive Pulsed Ultrasound Waving on Hydroxyapatite in Simulated Body Fluid. IEEE $11^{\text {th }}$ Inter. Confer. on Bioinf. Bioeng (BIBE) IEEE Computer Society; Taipei 125-129.

9. T Kokubo, H Takamada (2006) How useful is SBF in predicting in vivo bone bioactivity? Biomaterials, 27(15): 2907-2915.

10. A Tanaka, Y Sawada (1989) The Effect of the Pulse Ultrasonic Waves in the Dendritic Crystal Growth. The physical society of japan 19: 474

11. T Ichitsubo, E.Matsubara (2004) Ultrasound-induced crystallization around the glass transition temperature for Pd40Ni40P20 metallic glass. Acta Materialia 52(2): 423-429.

12. Ichitsubo T, Matsubara E, Anazawa $K$, Nishiyama $N$, Naito $M$ (2006) Low-temperature crystallization caused by ultrasound in Pd42.5Ni7.5Cu30P20 and Pd40Ni40P20 bulk metallic glasses. Mate Sci Eng 442 : 273-277.

13. Kokubo T, Miyaji F, Kim HM, Nakamura T (1996) Spontaneous formation of bonelike apatite layer on chemically treated titanium metals. J Am Cerami Soc 79(4): 1127-1129.

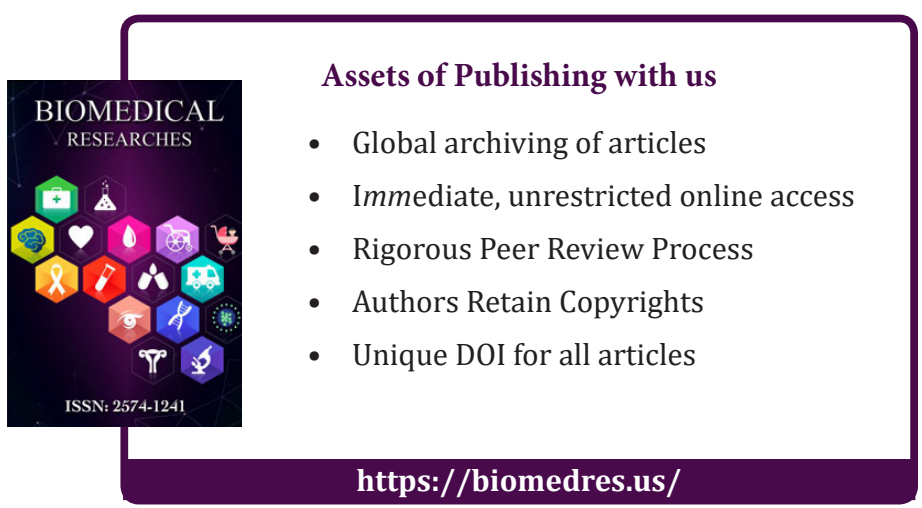

